\title{
A Mechanism Distinct from Highwire for the Drosophila Ubiquitin Conjugase Bendless in Synaptic Growth and Maturation
}

\author{
Smitha B. Uthaman, ${ }^{1}$ Tanja A. Godenschwege, ${ }^{2}$ and Rodney K. Murphey ${ }^{2}$ \\ ${ }^{1}$ Molecular and Cellular Biology Program, University of Massachusetts, Amherst, Massachusetts 01003, and ${ }^{2}$ Department of Biological Sciences, Florida \\ Atlantic University, Boca Raton, Florida 33431
}

\begin{abstract}
The signaling mechanisms that allow the conversion of a growth cone into a mature and stable synapse are yet to be completely understood. Ubiquitination plays key regulatory roles in synaptic development and may be involved in this process. Previous studies identified the Drosophila ubiquitin conjugase bendless (ben) to be important for central synapse formation, but the precise role it plays has not been elucidated. Our studies indicate that Ben plays a pivotal role in synaptic growth and maturation. We have determined that an incipient synapse is present with a high penetrance in ben mutants, suggesting that Ben is required for a developmental step after target recognition. We used cell-autonomous rescue experiments to show that Ben has a presynaptic role in synapse growth. We then harnessed the TARGET system to transiently express UAS (upstream activating sequence)-ben in a ben mutant background and identified a well defined critical period for Ben function in establishing a full-grown, mature synaptic terminal. We demonstrate that the protein must be present at a time point before but not during the actual growth process. We also provide phenotypic evidence demonstrating that Ben is not a part of the signal transduction pathway involving the well characterized ubiquitin ligase highwire. We conclude that Bendless functions as a novel developmental switch that permits the transition from axonal growth and incipient synapse formation to synaptic growth and maturation in the CNS.
\end{abstract}

Key words: giant fiber; Drosophila; synapse formation; ubiquitin; growth; maturation

\section{Introduction}

The formation, growth, and stabilization of synapses are crucial phenomena in the establishment of functional neural circuits. An important regulatory mechanism in synapse formation and function is ubiquitination, which refers to the posttranslational modification of a target protein by the addition of one (monoubiquitination) or more (multiubiquitination and polyubiquitination) adducts of the 76 amino acid ubiquitin polypeptide (Murphey and Godenschwege, 2002; DiAntonio and Hicke, 2004; Haas and Broadie, 2008). The transfer of ubiquitin to a substrate takes place through an enzymatic cascade involving a ubiquitin activating enzyme E1, a ubiquitin conjugating enzyme E2, and a ubiquitin ligase E3 (Hershko and Ciechanover, 1998). With regard to regulation of nervous system development, ubiquitin has been implicated in the regulation of axon guidance (Campbell and Holt, 2001), axonal pruning (Watts et al., 2003), synapse development (DiAntonio et al., 2001), neurotransmitter

\footnotetext{
Received June 28, 2008; accepted July 15, 2008.

This work was supported by National Institutes of Health (NIH) Grant R01-NS044609 (R.K.M.). T.A.G. was supported by NIH Grant R01 HD050725-01A1. We thank J. B. Thomas for kindly providing us with the bendless CDNA and the L. M. Schwartz laboratory, particularly Jeffery Kane and Chul Kim for molecular help and resources. We also thank P. Carrucio and X. Shan-Crofts for technical assistance in conducting the temperature shift experiments. For various fly stocks, we are grateful to the A. DiAntonio and C. Collins laboratories as well as the Bloomington Stock Center. Correspondence should be addressed to Dr. Rodney K. Murphey, Department of Biological Sciences, Sanson Science Building, Florida Atlantic University, Boca Raton, FL 33431. E-mail: rmurphey@fau.edu. D0I:10.1523/JNEUROSCI.2990-08.2008

Copyright $\odot 2008$ Society for Neuroscience $\quad 0270-6474 / 08 / 288615-09 \$ 15.00 / 0$
}

release (Wilson et al., 2002; Aravamudan and Broadie, 2003), number of postsynaptic receptors (Bedford et al., 2001; Buttner et al., 2001; Burbea et al., 2002; Patrick et al., 2003), and components of the postsynaptic density (Ehlers, 2003).

One of the first results that pointed to the ubiquitin system playing a role in the regulation of neuronal connectivity came from the analysis of the Drosophila mutant bendless (ben). ben was identified more than two decades ago in a behavioral screen for defects in the giant fiber system (GFS) (see Fig. $1 A, B$ ) that resulted in an altered escape response (Thomas and Wyman, 1982). Cloning of ben led to its identification as an E2 ubiquitin conjugase (Muralidhar and Thomas, 1993; Oh et al., 1994). The mutation was found to particularly affect the synapse between the giant fiber (GF) and the tergotrochanteral motorneuron (TTMn) as the GF axon, after making normal contact with the peripherally synapsing interneuron (PSI), fails to make the lateral "bend," which represents its large presynaptic terminal on the TTMn (Thomas and Wyman, 1984). Additional phenotypes were also reported in thoracic musculature (Edgecomb et al., 1993) and in the visual system (Muralidhar and Thomas, 1993; Oh et al., 1994).

Previous studies have suggested that Ben plays an important role in axon guidance or target recognition (Muralidhar and Thomas, 1993; Oh et al., 1994). However, the underlying mechanism by which Ben regulates the formation of a mature synapse is yet to be clarified. Our analyses of the ben mutant in the GF 
system using anatomical studies as well as specific approaches to determine the spatial and temporal aspects of gene function have given us novel evidence indicating that Ben plays an important role in synapse growth. Our studies suggest that Ben functions as an acute developmental switch that allows axonal growth to transition into synaptic growth, allowing for the establishment of a mature synaptic connection. To our knowledge, this is the first time that a component of the ubiquitin system has been shown to play the role of a key permissive factor that allows synaptic growth and maturation to take place, in contrast to, say, the ubiquitin ligase highwire (hiw) which can been characterized to actively regulate the growth process.

\section{Materials and Methods}

Drosophila stocks. All stocks were grown on standard medium at either $22^{\circ} \mathrm{C}$ or $25^{\circ} \mathrm{C}$ unless otherwise indicated. The following fly stocks were used: $b e n^{1}$, hereafter referred to as ben (Thomas and Wyman, 1984), ben deficiency (Bloomington stock 968, Df(1)HA92/FM7c), upstream activating sequence (UAS)-ben (second chromosome), ben; UAS-ben, UAS-green fluorescent protein (GFP)-ben (second chromosome), fat facets ( faf) EP(3)0381 (Rorth et al., 1998), hiw ${ }^{\mathrm{ND} 8}$ (Wan et al., 2000), and UAS-bsk $k^{\mathrm{DN}}$ (Weber et al., 2000). Three P[GAL] 4 drivers that express in the GF system were used: the A307 driver has strong presynaptic expression in the GF along with weaker expression in postsynaptic targets (Allen et al., 1998), c17 drives expression in the GF but not in its postsynaptic targets (Godenschwege et al., 2002), and ShakB-Gal4 drives expression only postsynaptically (Jacobs et al., 2000).

Generation of UAS-GFP-ben transgenic line. ben cDNA was cloned into the eGFPC1 vector with KpnI and SalI. The GFP-ben fragment was excised with NheI and SpeI and subcloned into the pUASt vector cut with $\mathrm{XbaI}$. Construct fidelity was verified by sequencing (Davis Sequencing, Davis, CA), and embryos were transformed at the Transgenic Drosophila Fly Core (Massachusetts General Hospital, Boston, MA).

Electrophysiology. Sharp electrode intracellular recordings from the TTM muscles were obtained from intact adult flies in a method similar to that described previously (Tanouye and Wyman, 1980). The physiological assay was modified and data were analyzed as described previously (Godenschwege et al., 2002).

Dye injections, imaging, and analysis. For anatomical analysis, the dissected CNS was mounted dorsal side up on a poly-lysine-coated slide. The preparation was immersed in saline and viewed with a $40 \times$ water immersion lens. The axons of the GFs are identified in the connective using differential interference contrast optics. A glass electrode (20-80 $\mathrm{M} \Omega$ ) containing $1 \%$ aqueous Lucifer yellow and backfilled with $3 \mathrm{M} \mathrm{LiCl}$ was used to impale the GF in the connective. The dye was injected into the GF by the passage of 3-5 $\mathrm{nA}$ of hyperpolarizing current using a Getting 5A Amplifier (Getting Instruments). Images in Figures 2 and 6 were obtained by taking maximum projections of Z-stack images taken using an advanced SPOT camera and MetaVue software. Deconvolution was performed on images using Autovisulize and Autoblur software version 9.3 (Autoquant). For UAS-GFP-ben localization in Figure 5, the image in $\boldsymbol{A}$ was taken using a coolSNAP HQ2 camera on a Nikon Eclipse FN1 microscope with $10 \times$ objective using NIS elements. Image was deconvolved with AutoquantX2 software (Media Cybernetics). Images in $\boldsymbol{B}-\boldsymbol{D}$ were taken on a Nikon Eclipse C1si confocal system with a $40 \times$ objective on a $90 \mathrm{i}$ microscope.

Ultrastructure. The adult CNS was dissected from ben flies that were electrophysiologically tested and wild-type (wt) controls. The CNS underwent prefixation in $2.5 \%$ glutaraldehyde for $24 \mathrm{~h}$ and was osmicated ( $1 \%$ osmium tetroxide) for $1 \mathrm{~h}$. Subsequent to dehydration, the CNS was embedded in Epon-Araldite. Ultrathin serial sections (50-60 nm) were taken and counterstained with $1 \%$ aqueous uranyl acetate and lead citrate. The sections were examined with a Jeol 100s electron microscope. The GF-TTMn synaptic region was photographed, and the negatives were scanned using a high-resolution flatbed scanner.

Temporal expression of UAS-ben using the TARGET system. The TARGET system (McGuire et al., 2003) was used to temporally express UASben in a ben mutant background. We crossed ben; UAS-ben flies to A307/
Cyo; tub-Gal80ts/TM6. The cross was set up at the permissive temperature (we found $22^{\circ} \mathrm{C}$ sufficient for the tub-Gal 80 to suppress A307 and restrict UAS-ben expression). Pupal day 0 (P0) pupae of ben/Y; A307/UAS-ben; tub-Gal80ts/+ were collected, and UAS-ben expression was induced for $24 \mathrm{~h}[\sim 16 \%$ of pupal development (PD)] by temperature shifts to $30^{\circ} \mathrm{C}$ at $\mathrm{P} 0, \mathrm{P} 0$ plus $6 \mathrm{~h}, \mathrm{P} 0$ plus $12 \mathrm{~h}, \mathrm{P} 0$ plus $18 \mathrm{~h}, \mathrm{P} 0$ plus $24 \mathrm{~h}, \mathrm{P} 0$ plus $48 \mathrm{~h}, \mathrm{P} 0$ plus $72 \mathrm{~h}, \mathrm{P} 0$ plus $96 \mathrm{~h}$, and P0 plus $120 \mathrm{~h}$. The TTM response was determined after eclosion in 2- to 3-d-old adults and compared with control specimens: ben/Y; A307/UAS-ben; TM6/+.

\section{Results}

\section{A residual synaptic connection is present in ben mutants}

We examined the ben phenotype by performing electrophysiological recordings from the TTM and found $88 \%$ of the responses to be mutant. A wild-type response from the muscle is defined as one with a response latency below $1 \mathrm{~ms}$ and which follows 100\% at $100 \mathrm{~Hz}$ stimulation. In accordance with previous studies, we found ben specimens to exhibit long, variable latencies and not follow 1:1 at high-frequency stimulation (Fig. $1 B$ ). To ascertain the penetrance of the mutant phenotype, we further crossed ben to a deficiency that spanned the entire ben gene and physiologically assayed offspring that were transheterozygous for ben and the deficiency. The physiology of the resultant transheterozygotes was $93 \%$ mutant (Table 1), which is not significantly different from the physiology of homozygous ben specimens as determined by a Fisher's exact test (two-tailed $p$ value $=1.000$ ).

For our anatomical analysis, we dissected the CNS out of the same ben specimens that had been physiologically tested and dye injected the GFs with Lucifer yellow, an anionic fluorescent dye that is small enough to pass through gap junctional contacts. In wild-type specimens, Lucifer yellow passes through the GF terminal into the TTMn $90-95 \%$ of the time depending on duration of the fill and the health of the specimen (Fig. 2A). We found transynaptic fills from the truncated GF terminal to the TTMn in $55 \%(n=22)$ of ben specimens (Fig. $2 B$ ). This indicated that there was a direct functional connection containing gap junctions between the GF and its target motor neuron. Because the ben mutation is an ethyl methane sulfonate-induced point mutant, it was possible that this synaptic contact pertains to a residual function of the mutant protein rather than being indicative of a null phenotype. To address this issue, we also performed Lucifer yellow dye injections in specimens that were transheterozygous for ben and the deficiency. We still saw transynaptic fills from the GF to the TTMn with a similar penetrance of $54 \%(n=16)$. To ensure that this residual synaptic connection seen in ben mutants was not just a case of delayed synaptogenesis or an initial stage of synaptic degeneration, we also analyzed ben mutants that were aged at $25^{\circ} \mathrm{C}$ for 2 weeks. Even in this case, the truncated GF terminal remained dye coupled with the TTMn.

Because the GF-TTMn synapse is a mixed electrical-chemical synapse (Blagburn et al., 1999; Allen and Murphey, 2007), we also wanted to determine whether components of the chemical synapse might be present in the terminals of ben mutants. We determined the localization of two synaptic vesicle markers, synaptotagmin and synaptobrevin, in the ben mutant by expressing GFPtagged constructs in the GFS. An example of synaptotagmin-GFP localization in a wild-type and a ben specimen is seen in Figure 2, $C$ and $D$, respectively. We could see fluorescent puncta of both proteins accumulate at the tips of the truncated terminal, suggesting the presence of synaptic vesicles $(n=18)$. We furthered this observation by performing ultrastructural analyses on the ben mutant and comparing the synaptic phenotype with what is seen in a wild-type specimen (Fig. $3 A, B$ ). We traced the GF to the synaptic region in three ben specimens and were able to isolate 


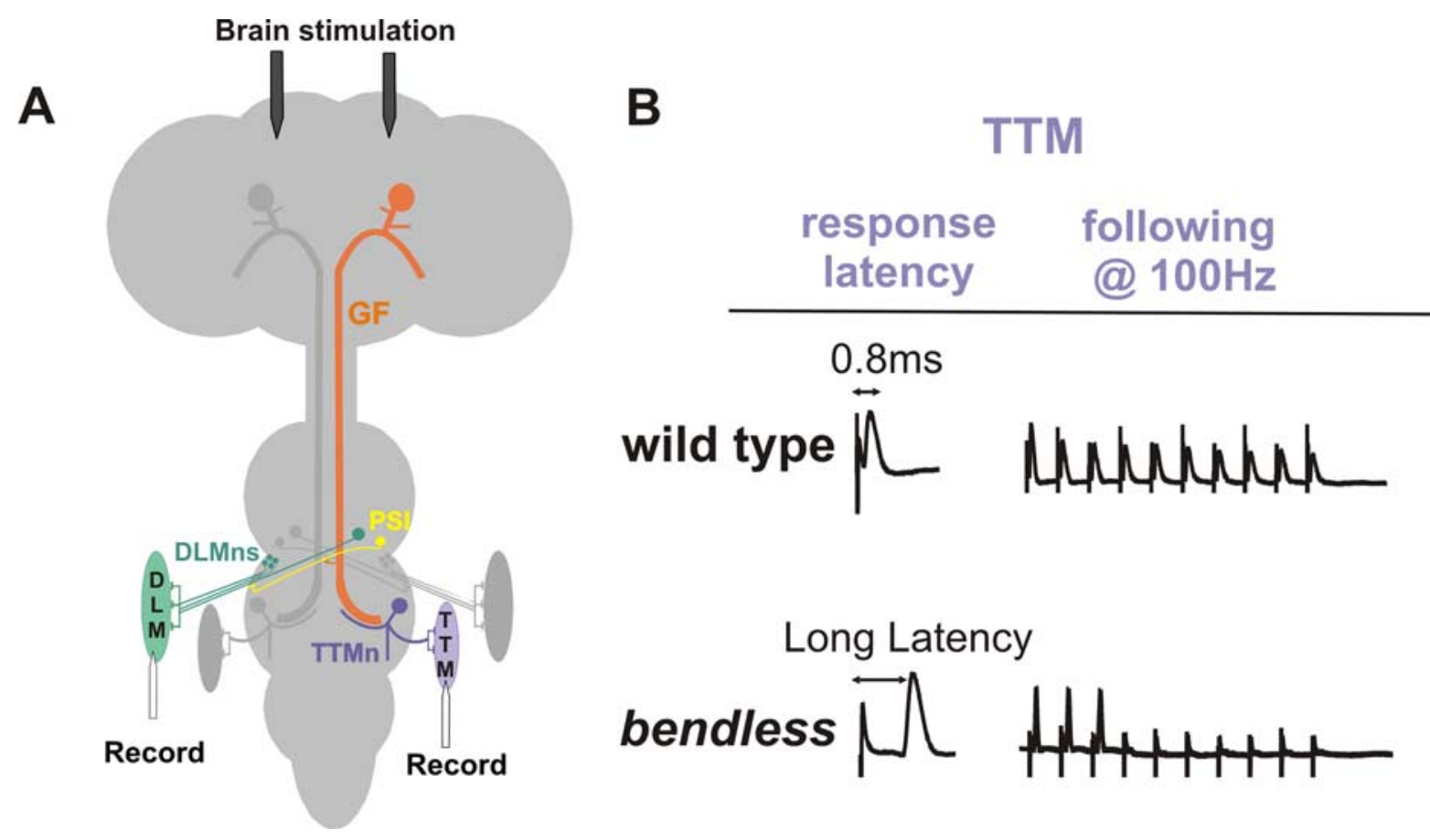

Figure 1. The giant fiber system. $\boldsymbol{A}$, Schematic of the GFS in the Drosophila CNS (modified from Godenschwege et al., 2006). The GFS is a well characterized neuronal circuit, and the mixed electrical- chemical synapse formed between the GF and the TTMn is an ideal setting to study the stages of synaptogenesis at a single-cell resolution both functionally and anatomically (King and Wyman, 1980; Blagburn et al., 1999). The image has been simplified to show the right half of the GF circuitry in color. The GF (orange) has its soma and dendrites in the brain, and its axons enter the second thoracic neuromere to make synaptic connections with the PSI (yellow) and the TTMn (blue). The PSI synapses onto the DLMn (green). The TTMn and the DLMn innervate the jump (TTM) and flight (DLM) muscles, respectively. Also indicated in the schematic are the stimulus and recording arrangement to obtain responses from the muscle. Although the recording from the right half of the circuitry is depicted, we obtain recordings from the muscle on both sides. Primary recordings are taken from the TTM, with recordings from the DLM taken to verify that the GF had indeed reached the target area. $\boldsymbol{B}$, Representative recordings from wild-type and bendless mutant adult flies. In wild-type specimens (top trace), the TTM response latency is $0.8 \mathrm{~ms}$ and the pathway is able to follow 1:1 at $100 \mathrm{~Hz}$ stimuli. Responses from bendless specimens (bottom trace) have characteristic long latencies ( $2 \mathrm{~ms}+$ ) and fail to follow $100 \%$ at $100 \mathrm{~Hz}$ stimulation.

Table 1. Spatial rescue of the physiological ben phenotype

\begin{tabular}{llll}
\hline Expression locus $^{a}$ & Genotype & $n$ & ${\text { Wild type in } \%^{b}}^{b}$ \\
\hline & +/y or +/+ & 40 & 100 \\
& ben/ben & 84 & 12 \\
ben/Def & 16 & 7 \\
Pre post & ben;UAS-ben/A307 & 22 & 100 \\
Pre & ben;UAS-ben/C17 & 24 & 83 \\
Pre & ben;UAS-bsk $/$ DN 17 & 16 & 6 \\
Pre & ben;UAS-ben/C17;UAS-ben & 32 & 91 \\
Pre & ben;UAS-ben/C42.2 & 14 & 20 \\
Post & ben;UAS-ben/Shak-B & 24 & 13 \\
Pre + post & $+/+; U A S-b e n / A 307$ & 24 & 100 \\
Pre + post & ben;A307/+;UAS-GFP-ben/+ & 16 & 93 \\
\hline
\end{tabular}

${ }^{a}$ Expression of UAS constructs presynaptically (pre) in the GF, postsynaptically (post) in the TTMn, or both.

${ }^{b}$ A wild-type synaptic connection is defined as a response latency $\leq 1 \mathrm{~ms}$ and the ability to follow stimuli one-to-one at $100 \mathrm{~Hz}$ when stimulated in the brain.

$n$, Number of GFs analyzed.

sections that show T-bars indicative of presynaptic release sites as well as close juxtaposition of the presynaptic and postsynaptic membrane with a single layer of vesicles indicative of gap junctional contact as described previously (Blagburn et al., 1999; Godenschwege et al., 2006) (Fig. 3C-F). The ultrastructural results are dramatic, and although the truncated GF tapers down to a much smaller axonal diameter, the essential synaptic features are still seen in the reduced contact region with the TTMn. These results suggest that an incipient chemical and gap junctional synapse is formed in a ben mutant background. Hence, Bendless does not seem to be required for nascent synapse formation but rather plays a role in synaptic growth and maturation.

\section{Bendless has a cell-autonomous presynaptic function}

We generated UAS-ben transgenic flies and conducted a series of rescue experiments to determine a spatial role for Bendless in the GFS. By driving UAS-ben expression in a ben mutant background under the control of A307, which is a strong driver that expresses in the GF and its postsynaptic target, we were able to completely rescue the Bendless phenotype physiologically and anatomically ( $100 \% \mathrm{wt} ; n=22$ ) (Table 1 and data not shown). Using the weaker presynaptic driver $\mathrm{cl}$, we were still able to get a significant rescue ( $83 \% \mathrm{wt} ; n=24)$ (Table 1 ), which improves with double the dosage of the UAS-ben transcript (91\% wt; $n=$ 32) (Table 1). In contrast, expression with the postsynaptic driver ShakB-Gal4 was unable to rescue the mutant phenotype (13\% wt; $n=24$ ) (Table 1). Our results confirm the previous observation that Bendless does indeed have a presynaptic role in synapse function (Oh et al., 1994) and additionally proves that it functions cell autonomously in the GFS.

Our anatomical studies led us to hypothesize that Bendless was involved in synaptic growth and maturation. Interestingly, driving expression of UAS-ben with A307 in a wild-type background did not disrupt the synapse physiologically $(100 \% \mathrm{wt} ; n=$ 24) (Table 1) or cause anatomical changes (data not shown). The absence of a gain-of-function phenotype, like an overgrown terminal, for Ben overexpression suggests that Bendless is not an effector molecule that is sufficient to induce synaptic growth on its own. We also expressed UAS-ben in a ben mutant background with the presynaptic c 42.2 driver, which turns on expression in the latter half of PD when the giant fiber would be undergoing additional growth after having made its synapse in wild-type specimens. We did not see any significant rescue of the ben phe- 

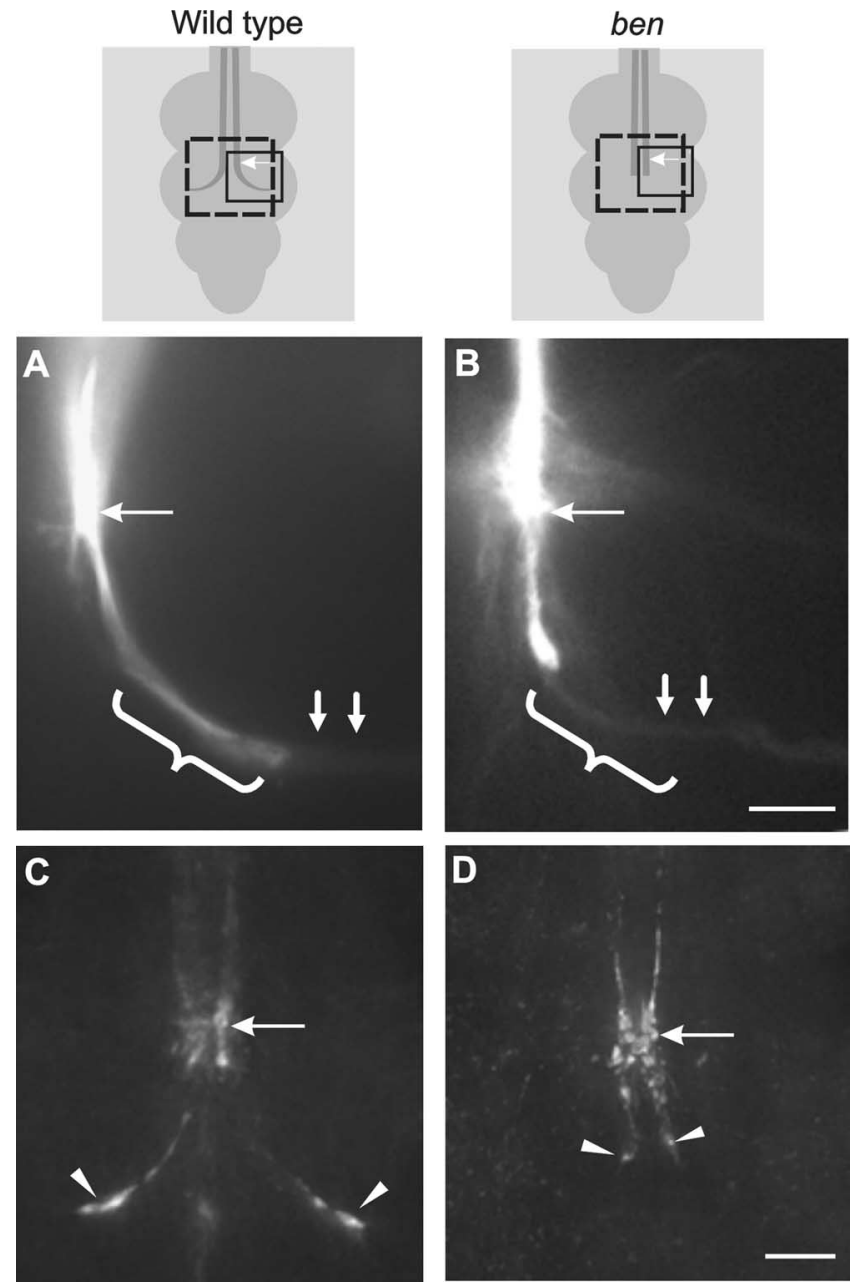

Figure 2. Analyses of the bendless incipient synapse. $\boldsymbol{A}$, Dye injection of the right GF in a wild-type specimen with Lucifer yellow. Solid line box in wild-type schematic indicates region of focus in the thoracic ganglion. The presynaptic terminal is indicated with a white bracket. A transynaptic fill from the GF to the TTMn is seen (vertical white arrows). $\boldsymbol{B}$, Dye injection of the right GF in a bendless specimen. Solid line box in ben schematic indicates region of focus. The presynaptic terminal is missing (white bracket), but a transynaptic fill from the truncated GF to the TTMn is still seen (vertical white arrows). C, Synaptotagmin-GFP localization in the GFs in the second thoracic neuromere of a wild-type specimen. Broken line box in wild-type schematic indicates region of focus in the thoracic ganglion. Strong fluorescence is seen the contact regions between the GF and PSI (white arrow) and the GF and TTMn (white arrowheads). $\boldsymbol{D}$, Synaptotagmin-GFP localization in a bendless specimen. Broken line box in ben schematic indicates region of focus. Fluorescent puncta are seen where the GF contacts the PSI (white arrow) and at the tips of the truncated GFs as well (white arrowheads). Scale bars, $10 \mu \mathrm{m}$ (in the respective top and panels). The horizontal white arrow in all panels serves as a point of reference indicating the region of contact between the GF and the PSI.

notype under these conditions (20\% wt; $n=13$ ) (Table 1$)$. This was intriguing and contrary to what might have been expected; Ben function was not required during the actual growth of the GF presynaptic terminal.

\section{Critical period for bendless before synaptic growth}

Because the expression of UAS-ben during later stages of PD by using the 442.2 driver did not rescue the ben mutant phenotype, we proceeded to determine more precisely the time point when protein function was necessary to make a normal synapse. Previous anatomical studies have delineated the various developmental stages of the GFS (Phelan et al., 1996; Allen et al., 1998) (Fig. $4 A$ ) and have also identified critical periods for synapse forma- tion (Murphey et al., 2003). We used the TARGET system (McGuire et al., 2004a,b) to regulate UAS-ben expression during different stages of GF development. Briefly, in the TARGET system, a temperature-sensitive variant of Gal80 (Gal80ts) is cloned behind a tubulin promoter and regulates Gal4 expression in a temperature-dependent manner. At a permissive temperature $\left(22^{\circ} \mathrm{C}\right)$, Gal80ts binds Gal4 and inhibits gene expression, whereas at a nonpermissive temperature $\left(30^{\circ} \mathrm{C}\right)$, the Gal 80 ts protein misfolds and does not bind Gal4 and gene expression is allowed to turn on.

In a ben mutant background, we turned on UAS-ben expression under the control of Gal80ts and the A307 driver by giving $24 \mathrm{~h}$ temperature shifts to nonpermissive temperature $\left(30^{\circ} \mathrm{C}\right)$ at a late larval stage (L3) and various stages of PD (Fig. $4 B$ ). The timeline of GF development that corresponds to these stages is indicated in the schematic (Fig. $4 A, B$ ). Although the duration of the temperature shift is indicated (Fig. $4 B$, red lines), it should be noted that transgene presence is likely to be seen with an $\sim 3 \mathrm{~h}$ delay and can persist for up to $\sim 12 \mathrm{~h}$ after the temperature shift as detailed in the original study (McGuire et al., 2004a). Comparing these results with internal controls, we found the period of maximum rescue to be when UAS-ben expression is turned on in the first 24 h of PD ( $80 \%$ wt; $n=132$ ) (Fig. $4 B$ ). The rescue ability decreases significantly for temperature shifts after P0 plus $12 \mathrm{~h}$ $(62 \% \mathrm{wt} ; n=34)$, and no rescue was seen after P0 plus $48 \mathrm{~h}(11 \%$ $\mathrm{wt} ; n=54)$. The time interval at which Ben is required to obtain any rescue of the mutant phenotype is indicated by the yellow highlight. We also performed temperature shifts of $48 \mathrm{~h}$ duration starting at $\mathrm{P} 0, \mathrm{P} 0$ plus $24 \mathrm{~h}, \mathrm{P} 0$ plus $48 \mathrm{~h}, \mathrm{P} 0$ plus $72 \mathrm{~h}, \mathrm{P} 0$ plus $96 \mathrm{~h}$, and $\mathrm{P} 0$ plus $120 \mathrm{~h}$ and still found a significant reduction in the rescue potential after P0 plus $24 \mathrm{~h}$ (data not shown). Hence, the critical period of UAS-ben expression is the first $24 \mathrm{~h}$ of $\mathrm{PD}$, a time interval earlier than the occurrence of synaptic growth (Fig. 4 , green highlight). This is rather interesting because the Ben protein is required for synaptic growth but need not be present during the growth event itself.

\section{Bendless localization is cytoplasmic and nuclear}

Ubiquitination is known to regulate synaptic function at both a protein level, by directly affecting signaling such as locally at the synapse, and a gene level, through the control of transcription factors (Murphey and Godenschwege, 2002; DiAntonio and Hicke, 2004). Hence, components of the ubiquitin system have been found to be expressed in both the nucleus and the cytosol. Sequence analysis points to Bendless being a cytoplasmic protein attributable to the lack of characterized nuclear localization motifs. To further characterize Bendless, we proceeded to determine its intracellular distribution. We generated a transgenic line containing UAS-ben with an N-terminal GFP fusion tag (UAS-GFPben). To verify that the GFP tag did not disrupt protein function, we expressed the construct under the control of the A307 driver in a ben mutant background and found that it was able to effect a robust rescue of the mutant phenotype (93\% wt; $n=16$ ) (Table $1)$. Given the functional rescue, we studied Ben protein localization by expressing UAS-GFP-ben in a wild-type background. We found that GFP-Ben is uniformly cytosolic throughout the entire neuron, inclusive of the GF axons, dendrites, and synaptic region (Fig. $5 A, D$ ). In addition to its ubiquitous cytosolic localization, we also find strong nuclear accumulation of GFP-Ben in cell bodies of the GF (Fig. 5B). Nuclear localization of GFP-Ben was seen in all subsets of neurons in which A307 Gal4 drives expression; an example of the dorsal longitudinal motorneuron (DLMn) cell body is seen in Figure 5C. These data highlight an 

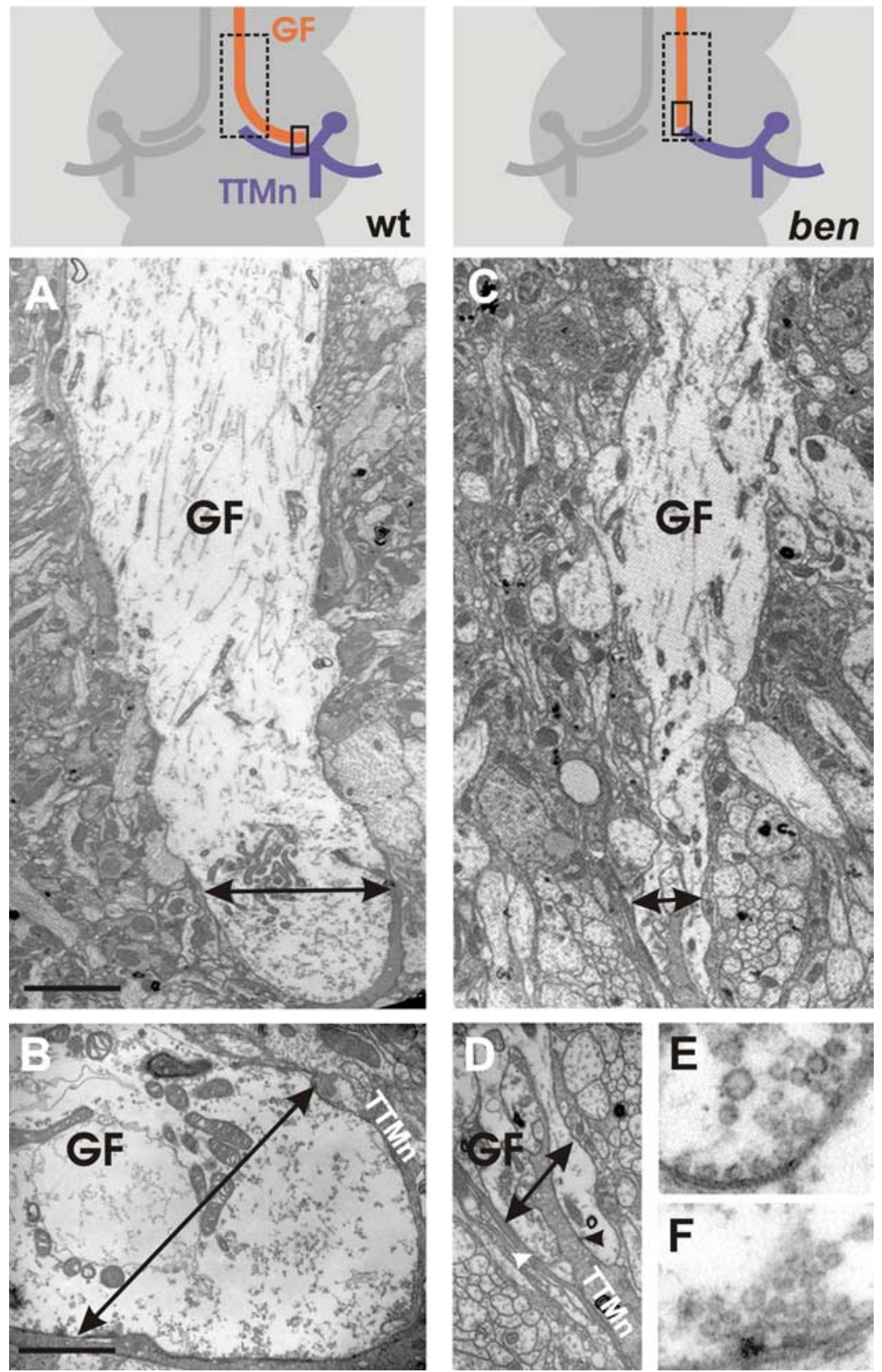

Figure 3. Ultrastructure of the presynaptic terminal in a bendless specimen. $A$, Electron micrograph showing the right $G F$ in the target area of a wild-type specimen. Broken line box in the wt schematic indicates the region of focus. The GF has a pronounced 5 $\mu \mathrm{m}$ diameter and is seen to contact the TTMn as indicated by the darker electron-dense region abutting the lighter GF tip. Scale bar: $A, C, 2 \mu \mathrm{m}$. $B$, Another section cut in the region marked by the solid box in the wt schematic showing a magnified view of the GF-TTMn contact region in a wild-type specimen. Note the expanded cross-sectional area of the presynaptic terminal. $C$, Electron micrograph showing the right GF in the target area of a ben specimen. Broken line box in schematic indicates the region of focus. The GF tapers in diameter and is seen to contact the TTMn. Scale bar: $\boldsymbol{B}, \boldsymbol{D}, 1 \mu \mathrm{m}$. $\boldsymbol{D}$, Another section showing a magnified view of the GF-TTMn contact region as pointed out by the solid box in the ben schematic. The TTMn interdigitates with the GF, and a vesicle release site with a T-bar (black arrowhead) is seen. An area with several vesicles is also indicated (white arrowhead). $\boldsymbol{E}$, Magnified image of a gap junctional contact with a characteristic single row of vesicles lining the membrane in ben. $\boldsymbol{F}$, Magnified image of a T-bar, the release site of a chemical synapse in ben.

important aspect of regulation whereby a molecule can have a precise temporal role affecting one particular synaptic connection despite having a ubiquitous spatial distribution.

\section{Bendless is in a distinct signaling pathway from Highwire}

In the established ubiquitination pathway, ubiquitin is transferred from an activating enzyme to a conjugating enzyme and finally onto a target substrate via a ubiquitin ligase that confers substrate specificity (Hershko and Ciechanover, 1998). Ben is a conjugating enzyme, and a possible candidate to be the downstream ligase for Ben in Drosophila is the ubiquitin ligase highwire. Hiw has been well characterized for regulating synaptic morphology at the Drosophila larval neuromuscular junction (NMJ) (Wan et al., 2000; DiAntonio et al., 2001; Wu et al., 2005, 2007; Collins et al., 2006). hiw mutants exhibit a dramatic increase in the number of synaptic branches and boutons along with an increase in synaptic span. NMJ synaptic development was found to be dependent on a balance of positive and negative regulators of ubiquitination. This balance is exemplified by the fact that hiw interacts with the deubiquitinating protease $f a f$, and overexpression of Faf results in a phenotype similar to hiw mutants (DiAntonio et al., 2001).

We wanted to determine whether ben and hiw acted through the same signaling cascade in establishing a central synapse by analyzing the mutants for phenotypic similarities. If Ben were the upstream conjugase partner of the ligase Hiw, then it follows that loss of Ben function should phenocopy loss of Hiw. We characterized the GFS, both physiologically and anatomically, in specimens carrying the hiw ${ }^{\mathrm{ND} 8}$ loss-of-function allele. Loss of hiw function did affect the GF-TTMn synapse physiologically as well as anatomically. Anatomically, we found ectopic axonal branches off the presynaptic terminal extending outside the target region in many specimens, but we did not find truncated GF terminals as seen in ben specimens $(n=30)$ (Table 2, Fig. 6B). Interestingly, we ascertained a similar anatomical result of axonal overgrowth with the overexpression of Faf in the GFS as well $(n=16)$ (Table 2), indicating that both loss-offunction hiw and gain-of-function Faf have similar effects on growth at a central synapse. Clearly, the ben mutant phenotype is distinctly different from the hiw and Faf phenotypes, with the former exhibiting synaptic undergrowth and the latter two exhibiting synaptic overgrowth.

To further confirm the distinct roles played by Ben and Hiw at a central synapse, we also looked at components of the hiw downstream signaling pathway. The downstream pathway of Drosophila hiw includes the mitogenactivated protein kinase kinase kinase wallenda (wnd), the Jun kinase (JNK) basket (bsk), and the D-fos transcription factor. At the NMJ, the inhibition of JNK signaling by the expression of a UAS- $b s k^{\mathrm{DN}}$ construct was found to suppress the hiw phenotype (Collins et al., 2006). We expressed UAS-bsk ${ }^{\mathrm{DN}}$ in a ben mutant background and did not find the ben phenotype to be suppressed 


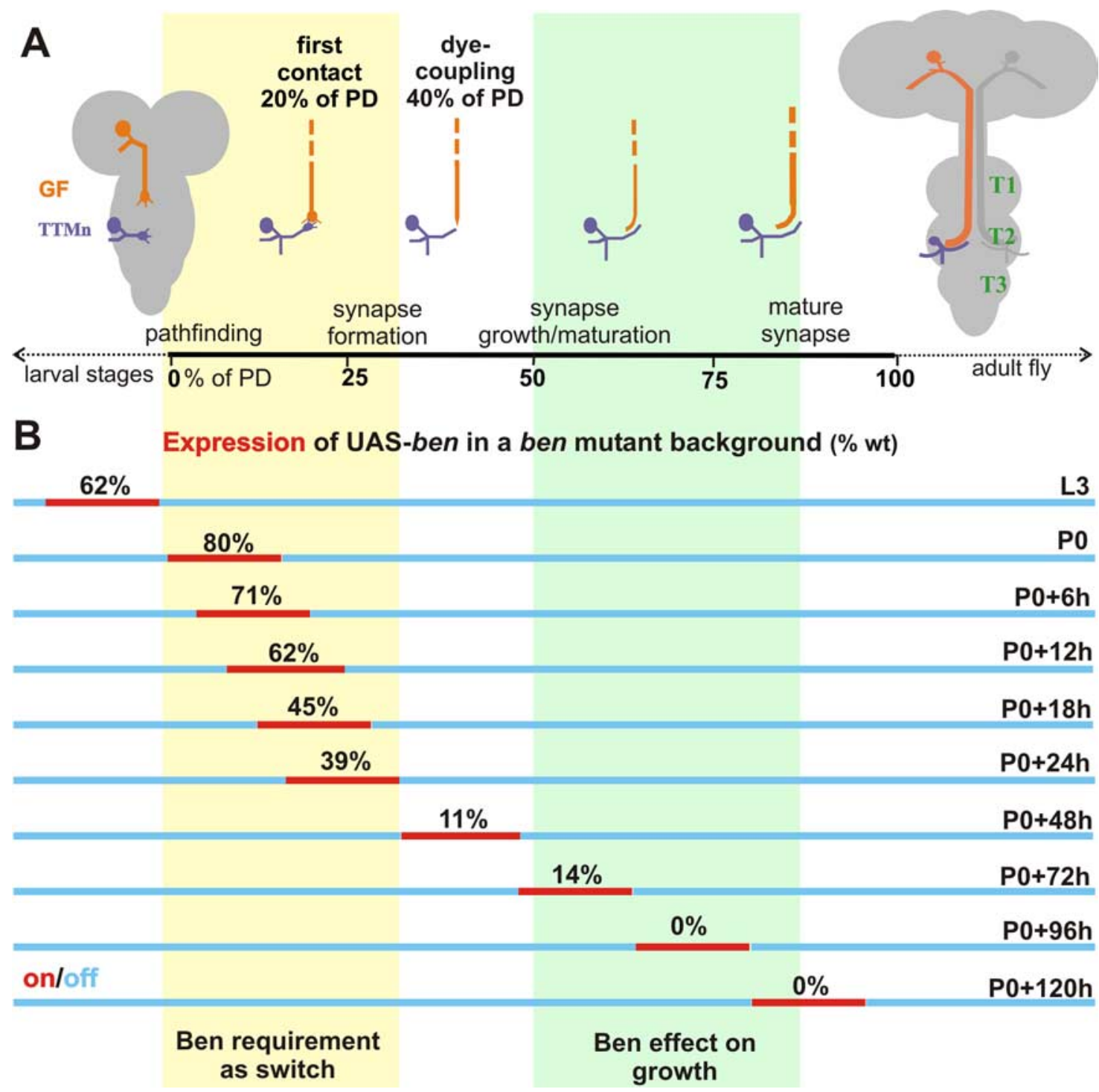

Figure 4. Critical period for Bendless function. A, Schematic showing the various phases of giant synapse assembly during PD (modified from Godenschwege et al., 2006). The TTMn dendrites grow into the target area of the second thoracic neuromere before the arrival of GF axons. The GF initiates growth in the late larval stage and reaches the target area to make initial contact with the TTMn dendrite by $20 \%$ of PD. This is followed by a phase of synaptogenesis ( $25-50 \%$ of PD) during which the GF dye couples with the TTMn at $\sim 40 \%$ of PD and elaborates a lateral "bend" establishing a presynaptic terminal (Phelan et al., 1996; Allen et al., 1998; Jacobs et al., 2000). After $50 \%$ of PD, the synapse continues to grow and stabilize to become a fully mature synapse by the time of eclosion. The green highlight indicates the period of synaptic growth, and the yellow highlight indicates the time period when Ben function is required to affect a rescue of the mutant phenotype. $\boldsymbol{B}$, Representation of temperature shifts (TS) given to specimens of genotype ben/Y;A307/UAS-ben; Gal80ts/+ at various stages of PD using the TARGET system. Specimens were raised at the permissive temperature of $22^{\circ} \mathrm{C}$ (blue line indicating that gene expression is " off") and then placed at a nonpermissive temperature of $30^{\circ} \mathrm{C}$ for $24 \mathrm{~h}$ (red line indicating that gene expression is "on"). A $24 \mathrm{~h}$, temperature shift represents $\sim 16 \%$ of PD. The percentages over the red lines indicate the percentage of wild-type responses (response latency $\leq 1 \mathrm{~ms}$ and the ability to follow stimuli one-to-one at $100 \mathrm{~Hz}$ when stimulated in the brain) from specimens tested as adults. The number of specimens tested ( $\mathrm{n}$ ) are as follows: $\mathrm{no}$ TS (68), L3 (8), P0 (132), P0 plus $6 \mathrm{~h}$ (31), P0 plus $12 \mathrm{~h}$ (34), P0 plus $18 \mathrm{~h}$ (22), PO plus $24 \mathrm{~h}$ (64), P0 plus $48 \mathrm{~h}$ (54), PO plus $72 \mathrm{~h}$ (36), P0 plus $96 \mathrm{~h}$ (74), and P0 plus $120 \mathrm{~h}$ (13). Control specimens of the genotype ben/Y; A307/UAS-ben; TM6/ $+(n=$ 210) were also tested under the same paradigm at different stages of PD, and responses were found to be $82-100 \%$ wild type.

(Table 1). Overall, our experiments strongly suggest that Ben and Hiw function in distinct pathways to regulate central synapse growth. A detailed description of the hiw phenotype, additional analysis of its function, and subsequent characterization of its downstream signaling cascade at a central synapse will be performed in a separate study.

\section{Discussion}

\section{Bendless and Highwire}

The results from this study have given us new insights into how ubiquitin system components establish functional synaptic connections. The temporal analysis of Bendless has been critical in illustrating its role as a developmental "switch" in converting a growth cone into a mature synapse. As mentioned previously, the ben mutation is the result of a single amino acid change in the conserved catalytic core of the conjugase domain of the protein. This highlights the fact that the conjugase activity of the protein is necessary for the observed synaptic phenotype. Analysis of synaptic growth in Drosophila has primarily been done at the peripheral synapse of the NMJ (Laviolette et al., 2005; Goold and Davis, 2007; Fulga and Van Vactor, 2008; Liebl et al., 2008; Schwenkert et al., 2008; Seabrooke and Stewart, 2008). Components of the ubiquitin system, such as the ubiquitin ligase hiw, the deubiquitinating protease faf, and the synapseassociated E3 ligase PDZRN3, are known to play important roles in the growth and function of the fly NMJ (DiAntonio et al., 2001; 


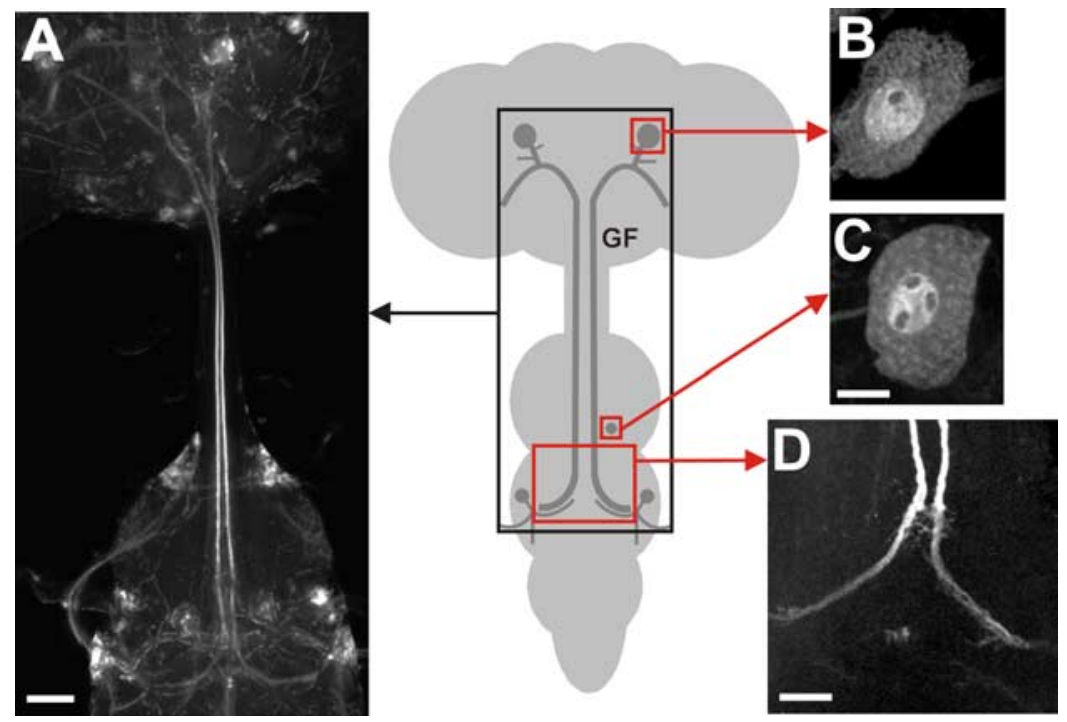

Figure 5. Localization of Bendless. $\boldsymbol{A}$, Deconvolved image of a specimen expressing UAS-GFP-ben under the control of the A307 driver at $10 X$. The specified region is indicated by the solid black box in the schematic. $B$, Confocal image of GF cell body in the brain showing diffuse localization of UAS-GFP-ben in the cytoplasm as well as intense nuclear staining. Solid top red line box in schematic indicates GF cell body position in brain. , Confocal image of DLMn cell body in the thoracic ganglion showing cytoplasmic and nuclear localization of GFP-Ben. Solid middle red line box in schematic indicates DLMn cell body position in thoracic ganglion. $\boldsymbol{D}$, Confocal image showing the uniform localization of UAS-GFP-ben in the presynaptic terminal. Solid bottom red line box in schematic indicates the specified location in the thoracic ganglion. Scale bars: $\boldsymbol{A}, 25 \mu \mathrm{m} ; \boldsymbol{B}, \boldsymbol{C}, 5 \mu \mathrm{m} ; \boldsymbol{D}, 10 \mu \mathrm{m}$.

Table 2. Anatomical analysis of Hiw and Faf in the GFS

\begin{tabular}{lllll}
\hline & & \% of specimens with & \\
\cline { 3 - 5 } Genotype & $n$ & Synaptic terminal present & bendless-like terminal & Extra axonal branches \\
\hline hiw/hiw & 30 & 100 & 0 & 47 \\
A307-ULZ; faf EP(3)381 & 16 & 100 & 0 & 38 \\
\hline
\end{tabular}

$n$, Number of GFs analyzed.

McCabe et al., 2004; Wu et al., 2005; Collins et al., 2006; Lu et al., 2007). Significant studies have been performed with particular regard to the conserved family of hiw ubiquitin ligases. In Drosophila, hiw functions as a negative regulator of synapse development as mutants exhibit dramatic synaptic overgrowth at the larval NMJ (Wan et al., 2000). In Caenorhabditis elegans, loss of function of the hiw homolog rpm-1 results in multiple phenotypes at the NMJ as well as in the CNS (Schaefer et al., 2000; Zhen et al., 2000). At the NMJ, some NMJs exhibit enlarged presynaptic terminals containing multiple active zones, whereas others contain underdeveloped or absent presynaptic terminals (Zhen et al., 2000). In the worm mechanosensory circuit, the sensory neurons were found to retract synaptic branches, extend ectopic axons, and fail to accumulate synaptic vesicles, whereas some of the motor neurons exhibited phenotypes such as altered synaptic organization, branching, and overgrowth (Schaefer et al., 2000). Downstream signaling components have been isolated for both hiw and rpm-1 in Drosophila and C. elegans, respectively, and a number of conserved elements have been identified (Schaefer et al., 2000; Nakata et al., 2005; Collins et al., 2006; Grill et al., 2007; Abrams et al., 2008; Li et al., 2008). Mutations in homologs of hiw in zebrafish and mice are also known to cause a variety of synaptic disruptions (Burgess et al., 2004; D’Souza et al., 2005).

Ben and Hiw play distinct roles in synapse growth. In our study, we have been able to analyze the novel roles played by these ubiquitin system components at the GFS central synapse. We have shown that ben and hiw loss of function result in very different phenotypes, with ben specimens exhibiting synaptic un- dergrowth and hiw specimens exhibiting synaptic overgrowth. We also show that Ben function does not involve JNK, a well characterized downstream signaling partner identified for Hiw in Drosophila. It is also interesting to compare and contrast the role Hiw plays at a peripheral synapse with the role Ben plays at a central synapse. hiw mutants exhibit a presynaptic overgrowth phenotype at the NMJ, whereas ben mutants exhibit a reduction in presynaptic growth in the CNS. Also, Hiw does not localize to the nucleus and was found to regulate synaptic growth throughout development, whereas Ben has nuclear as well as cytosolic localization and only functions in a critical time period. Finally, Hiw activity is associated with the bone morphogenetic protein (BMP) retrograde signaling pathway that is known to be dependent on the retrograde motor (McCabe et al., 2004). We found no evidence that Bendless function is dependent on the retrograde motor (our unpublished data). All these data underline the fact that there are distinct targets for the ubiquitination cascades involving Ben and Hiw.

\section{Bendless is required for synaptic growth and maturation}

Functional neuronal circuits are established through a series of events: neurite outgrowth, axon guidance, target recognition, synapse formation, and synaptic growth and maturation. When the bendless mutant was originally characterized, Ben was thought to play an important role in either axon guidance or target recognition (Muralidhar and Thomas, 1993; Oh et al., 1994). Our analysis of the ben mutant clearly shows that Ben has an important role in synaptic growth. A number of specimens exhibit dye coupling between the GF and the motorneuron dendrite demonstrating that an incipient synapse is still formed (Fig. $2 \mathrm{~B}$ ) and that the mutant phenotype arises from a failure of this immature connection to grow into a mature synapse. In addition, we also shown that both gap junctional and chemical components are present at ben mutant terminals with synaptic vesicle marker localization as well as ultrastructural analyses (Figs. 2D, $3 C-F)$.

The current view of synapse formation is that a nascent synapse can be rapidly assembled from material present in a growth cone in prepackaged vesicles and packets (Roos and Kelly, 2000; Ziv and Garner, 2004). After this primary rapid assembly of a nascent synapse, a secondary slower growth and maturation process takes place to result in a stable mature synapse. An insightful study on the Drosophila kinesin immaculate connections (imac) has shown it to be a permissive regulator of presynaptic maturation at the larval NMJ (Pack-Chung et al., 2007). Imac was found to be involved in the anterograde transport of synaptic vesicle precursors to the tip of the growth cone, an initial stage of synaptogenesis. In ben specimens, synaptic vesicles are still transported all the way down to the tip of the truncated terminal as evidenced by the localization of GFP-tagged synaptotagmin and synaptobrevin (Fig. 2D). Our data strongly suggest that the ben mutant 
phenotype is resultant at a point after synaptic vesicular transport. Hence, we conclude that the bendless terminal is an incipient synapse that fails to grow and mature and that Ben is a permissive regulator whose function is required for the initiation of a secondary process in presynaptic growth.

\section{Bendless as a developmental switch}

It is counterintuitive that, although Bendless is required for synaptic growth and maturation, our data show that it is not required during the growth process. This highlights the important role Ben plays as a developmental switch. Transient expression of UAS-ben before the growth of the presynaptic terminal was sufficient to rescue the ben phenotype anatomically and physiologically, but expression during the growth period had no effect (Fig. 4). This suggests that Bendless is not involved in the actual growth process but rather has to be present in advance to alter signaling and initiate changes that allow growth to take place. Here it is essential to differentiate between axonal and synaptic growth, because it has been determined previously that axonal growth is unaffected in ben mutants (Muralidhar and Thomas, 1993). Hence, Ben function is required to permit axonal growth to switch to synaptic growth.

The molecules in the signaling pathway of this novel mechanism remain to be further investigated. In conclusion, tight spatial and temporal control of synaptic connectivity in the nervous system is undoubtedly crucial to normal function. Determining how exactly Bendless regulates the formation of a mature synapse will give us future novel insights into this phenomenon.

\section{References}

Abrams B, Grill B, Huang X, Jin Y (2008) Cellular and molecular determinants targeting the Caenorhabditis elegans PHR protein RPM-1 to perisynaptic regions. Dev Dyn 237:630-639.

Allen MJ, Murphey RK (2007) The chemical component of the mixed GFTTMn synapse in Drosophila melanogaster uses acetylcholine as its neurotransmitter. Eur J Neurosci 26:439-445.

Allen MJ, Drummond JA, Moffat KG (1998) Development of the giant fiber neuron of Drosophila melanogaster. J Comp Neurol 397:519-531.

Aravamudan B, Broadie K (2003) Synaptic Drosophila UNC-13 is regulated by antagonistic G-protein pathways via a proteasome-dependent degradation mechanism. J Neurobiol 54:417-438.

Bedford FK, Kittler JT, Muller E, Thomas P, Uren JM, Merlo D, Wisden W, Triller A, Smart TG, Moss SJ (2001) GABA(A) receptor cell surface number and subunit stability are regulated by the ubiquitin-like protein Plic-1. Nat Neurosci 4:908-916.

Blagburn JM, Alexopoulos H, Davies JA, Bacon JP (1999) Null mutation in shaking-B eliminates electrical, but not chemical, synapses in the Drosophila giant fiber system: a structural study. J Comp Neurol 404:449-458.

Burbea M, Dreier L, Dittman JS, Grunwald ME, Kaplan JM (2002) Ubiquitin and AP180 regulate the abundance of GLR-1 glutamate receptors at postsynaptic elements in C. elegans. Neuron 35:107-120.

Burgess RW, Peterson KA, Johnson MJ, Roix JJ, Welsh IC, O'Brien TP (2004) Evidence for a conserved function in synapse formation reveals Phrl as a candidate gene for respiratory failure in newborn mice. Mol Cell Biol 24:1096-1105.

Büttner C, Sadtler S, Leyendecker A, Laube B, Griffon N, Betz H, Schmalzing G (2001) Ubiquitination precedes internalization and proteolytic cleav- age of plasma membrane-bound glycine receptors. J Biol Chem 276:42978-42985.

Campbell DS, Holt CE (2001) Chemotropic responses of retinal growth cones mediated by rapid local protein synthesis and degradation. Neuron 32:1013-1026.

Collins CA, Wairkar YP, Johnson SL, DiAntonio A (2006) Highwire restrains synaptic growth by attenuating a MAP kinase signal. Neuron 51:57-69.

DiAntonio A, Hicke L (2004) Ubiquitin-dependent regulation of the synapse. Annu Rev Neurosci 27:223-246.

DiAntonio A, Haghighi AP, Portman SL, Lee JD, Amaranto AM, Goodman CS (2001) Ubiquitination-dependent mechanisms regulate synaptic growth and function. Nature 412:449-452.

D’Souza J, Hendricks M, Le Guyader S, Subburaju S, Grunewald B, Scholich K, Jesuthasan S (2005) Formation of the retinotectal projection requires Esrom, an ortholog of PAM (protein associated with Myc). Development 132:247-256.

Edgecomb RS, Ghetti C, Schneiderman AM (1993) Bendless alters thoracic musculature in Drosophila. J Neurogenet 8:201-219.

Ehlers MD (2003) Activity level controls postsynaptic composition and signaling via the ubiquitin-proteasome system. Nat Neurosci 6:231-242.

Fulga TA, Van Vactor D (2008) Synapses and growth cones on two sides of a highwire. Neuron 57:339-344.

Godenschwege TA, Simpson JH, Shan X, Bashaw GJ, Goodman CS, Murphey RK (2002) Ectopic expression in the giant fiber system of Drosophila reveals distinct roles for roundabout (Robo), Robo2, and Robo3 in dendritic guidance and synaptic connectivity. J Neurosci 22:3117-3129.

Godenschwege TA, Kristiansen LV, Uthaman SB, Hortsch M, Murphey RK (2006) A conserved role for Drosophila Neuroglian and human L1-CAM in central-synapse formation. Curr Biol 16:12-23.

Goold CP, Davis GW (2007) The BMP ligand Gbb gates the expression of synaptic homeostasis independent of synaptic growth control. Neuron 56:109-123.

Grill B, Bienvenut WV, Brown HM, Ackley BD, Quadroni M, Jin Y (2007) C. elegans RPM-1 regulates axon termination and synaptogenesis through the Rab GEF GLO-4 and the Rab GTPase GLO-1. Neuron 55:587-601.

Haas KF, Broadie K (2008) Roles of ubiquitination at the synapse. Biochim Biophys Acta, in press.

Hershko A, Ciechanover A (1998) The ubiquitin system. Annu Rev Biochem 67:425-479.

Jacobs K, Todman MG, Allen MJ, Davies JA, Bacon JP (2000) Synaptogen- 
esis in the giant-fibre system of Drosophila: interaction of the giant fibre and its major motorneuronal target. Development 127:5203-5212.

King DG, Wyman RJ (1980) Anatomy of the giant fibre pathway in Drosophila. I. Three thoracic components of the pathway. J Neurocytol 9:753-770.

Laviolette MJ, Nunes P, Peyre JB, Aigaki T, Stewart BA (2005) A genetic screen for suppressors of Drosophila NSF2 neuromuscular junction overgrowth. Genetics 170:779-792.

Li H, Kulkarni G, Wadsworth WG (2008) RPM-1, a Caenorhabditis elegans protein that functions in presynaptic differentiation, negatively regulates axon outgrowth by controlling SAX-3/robo and UNC-5/UNC5 activity. J Neurosci 28:3595-3603.

Liebl FL, Wu Y, Featherstone DE, Noordermeer JN, Fradkin L, Hing H (2008) Derailed regulates development of the Drosophila neuromuscular junction. Dev Neurobiol 68:152-165.

Lu Z, Je HS, Young P, Gross J, Lu B, Feng G (2007) Regulation of synaptic growth and maturation by a synapse-associated $\mathrm{E} 3$ ubiquitin ligase at the neuromuscular junction. J Cell Biol 177:1077-1089.

McCabe BD, Hom S, Aberle H, Fetter RD, Marques G, Haerry TE, Wan H, O'Connor MB, Goodman CS, Haghighi AP (2004) Highwire regulates presynaptic BMP signaling essential for synaptic growth. Neuron 41:891-905.

McGuire SE, Le PT, Osborn AJ, Matsumoto K, Davis RL (2003) Spatiotemporal rescue of memory dysfunction in Drosophila. Science 302:1765-1768.

McGuire SE, Mao Z, Davis RL (2004a) Spatiotemporal gene expression targeting with the TARGET and gene-switch systems in Drosophila. Sci STKE 2004:16.

McGuire SE, Roman G, Davis RL (2004b) Gene expression systems in Drosophila: a synthesis of time and space. Trends Genet 20:384-391.

Muralidhar MG, Thomas JB (1993) The Drosophila bendless gene encodes a neural protein related to ubiquitin-conjugating enzymes. Neuron 11:253-266.

Murphey RK, Godenschwege TA (2002) New roles for ubiquitin in the assembly and function of neuronal circuits. Neuron 36:5-8.

Murphey RK, Froggett SJ, Caruccio P, Shan-Crofts X, Kitamoto T, Godenschwege TA (2003) Targeted expression of shibire ts and semaphorin 1a reveals critical periods for synapse formation in the giant fiber of Drosophila. Development 130:3671-3682.

Nakata K, Abrams B, Grill B, Goncharov A, Huang X, Chisholm AD, Jin Y (2005) Regulation of a DLK-1 and p38 MAP kinase pathway by the ubiquitin ligase RPM-1 is required for presynaptic development. Cell 120:407-420.

Oh CE, McMahon R, Benzer S, Tanouye MA (1994) bendless, a Drosophila gene affecting neuronal connectivity, encodes a ubiquitin-conjugating enzyme homolog. J Neurosci 14:3166-3179.

Pack-Chung E, Kurshan PT, Dickman DK, Schwarz TL (2007) A Drosophila kinesin required for synaptic bouton formation and synaptic vesicle transport. Nat Neurosci 10:980-989.
Patrick GN, Bingol B, Weld HA, Schuman EM (2003) Ubiquitin-mediated proteasome activity is required for agonist-induced endocytosis of GluRs. Curr Biol 13:2073-2081.

Phelan P, Nakagawa M, Wilkin MB, Moffat KG, O'Kane CJ, Davies JA, Bacon JP (1996) Mutations in shaking-B prevent electrical synapse formation in the Drosophila giant fiber system. J Neurosci 16:1101-1113.

Roos J, Kelly RB (2000) Preassembly and transport of nerve terminals: a new concept of axonal transport. Nat Neurosci 3:415-417.

Rørth P, Szabo K, Bailey A, Laverty T, Rehm J, Rubin GM, Weigmann K, Milán M, Benes V, Ansorge W, Cohen SM (1998) Systematic gain-offunction genetics in Drosophila. Development 125:1049-1057.

Schaefer AM, Hadwiger GD, Nonet ML (2000) rpm-1, a conserved neuronal gene that regulates targeting and synaptogenesis in C. elegans. Neuron 26:345-356.

Schwenkert I, Eltrop R, Funk N, Steinert JR, Schuster CM and Scholz H (2008) The hangover gene negatively regulates bouton addition at the Drosophila neuromuscular junction. Mech Dev 125:700-711.

Seabrooke S, Stewart BA (2008) Moesin helps to restrain synaptic growth at the Drosophila neuromuscular junction. Dev Neurobiol 68:379-391.

Tanouye MA, Wyman RJ (1980) Motor outputs of giant nerve fiber in Drosophila. J Neurophysiol 44:405-421.

Thomas JB, Wyman RJ (1982) A mutation in Drosophila alters normal connectivity between two identified neurones. Nature 298:650-651.

Thomas JB, Wyman RJ (1984) Mutations altering synaptic connectivity between identified neurons in Drosophila. J Neurosci 4:530-538.

Wan HI, DiAntonio A, Fetter RD, Bergstrom K, Strauss R, Goodman CS (2000) Highwire regulates synaptic growth in Drosophila. Neuron 26:313-329.

Watts RJ, Hoopfer ED, Luo L (2003) Axon pruning during Drosophila metamorphosis: evidence for local degeneration and requirement of the ubiquitin-proteasome system. Neuron 38:871-885.

Weber U, Paricio N, Mlodzik M (2000) Jun mediates Frizzled-induced $\mathrm{R} 3 / \mathrm{R} 4$ cell fate distinction and planar polarity determination in the Drosophila eye. Development 127:3619-3629.

Wilson SM, Bhattacharyya B, Rachel RA, Coppola V, Tessarollo L, Householder DB, Fletcher CF, Miller RJ, Copeland NG, Jenkins NA (2002) Synaptic defects in ataxia mice result from a mutation in Usp14, encoding a ubiquitin-specific protease. Nat Genet 32:420-425.

Wu C, Wairkar YP, Collins CA, DiAntonio A (2005) Highwire function at the Drosophila neuromuscular junction: spatial, structural, and temporal requirements. J Neurosci 25:9557-9566.

Wu C, Daniels RW, DiAntonio A (2007) DFsn collaborates with Highwire to down-regulate the Wallenda/DLK kinase and restrain synaptic terminal growth. Neural Develop 2:16.

Zhen M, Huang X, Bamber B, Jin Y (2000) Regulation of presynaptic terminal organization by C. elegans RPM-1, a putative guanine nucleotide exchanger with a RING-H2 finger domain. Neuron 26:331-343.

Ziv NE, Garner CC (2004) Cellular and molecular mechanisms of presynaptic assembly. Nat Rev Neurosci 5:385-399. 